\title{
Relationship between Systemic Adaptation to Physical Effort and Plasma Potassium in Untrained and Trained Andalusian and Angloarabian Horses
}

\author{
Ana MUÑOZ ${ }^{1}$, Gristina RIBER ${ }^{2}$, Katiuska SATUÉ ${ }^{1}$, Raquel G. LUCAS ${ }^{1}$ and Milagros BENITO ${ }^{1}$ \\ ${ }^{1}$ Department of Animal Medicine and Surgery, Cardenal Herrera - CEU University, 46113 Moncada, Valencia, \\ ${ }^{2}$ Department of Animal Medicine and Surgery, Faculty of Veterinary Medicine, Córdoba University, Córdoba, \\ Spain
}

Plasma K accumulation during exercise results from the balance between exchange through biological membranes (mainly muscle fibres and erythrocytes), distribution to other tissues and the haemoconcentration. In the present study, the effect of exercise and training on plasma $K$ concentrations and its relationships with other physiological variables have been analysed in two equine breeds. Twenty male Andalusian (AN) and ten Angloarabian (AA) horses, 7 females and 3 males, were subjected to two standardised exercise tests, composed of four workloads, before and after training. Heart rate (HR) was monitored and venous blood was withdrawn at rest, before each exercise level and during recovery. The following parameters were analysed: packed cell volume (PCV), plasma $K$, lactate (LA) and total protein (TPP). Furthermore, the horses were filmed and three kinematic parameters were studied: stride duration (SD), frequency (SF) and length (SL). Exercise induced an increase in $K$ from 6 and $8 \mathrm{~m} / \mathrm{sec}$ in $A A$ and $A N$ horses respectively, a steady-state until the end of the exercise and a decrease after 2 min of recuperation. Some interbreed differences existed, with higher $K$ levels in the AN horses, due to the higher relative exercise intensity, stride frequency and haemoconcentration. K was correlated with HR, PCV, TPP, SL, SD and SF. Training caused a decrease in $K$ in AN, but not in AA horses. Plasma K seems to be a good indicator of the physical effort intensity, fitness and training degrees, but it was not related to the magnitude of the glycolytic response to exercise.

Key words: exercise, horses, lactate, potassium, training
J. Equine Sci.

Vol. 14, No. 1

pp. 13-22, 2003
An action potential causes a $\mathrm{K}$ efflux at a rate of $0.3-$ $0.9 \mathrm{nmol} \mathrm{K} /$ impulse per gram muscle weight. During high intensity exercise, when millions of muscle cells are excited repeatedly, plasma $\mathrm{K}$ accumulation may reach values above $8 \mathrm{mmol} / l[11,33]$. The $\mathrm{K}$ response to muscular contraction is an initial rapid increase in plasma concentrations followed by a slow increase until steady-state values are reached. The extent of the increase is generally related to the intensity of exercise, although inter specific differences have been reported. The $\mathrm{K}$ response to maximal speed is therefore greater in horses than in man and calves $[8,28,29]$, with

This article was accepted March 20, 2003.

${ }^{*}$ Corresponding author. e-mail: amjuzado@uch.ceu.es prolonged submaximal exercise plasma $\mathrm{K}$ concentrations usually decreasing to below behind resting levels. This fact has been documented in humans [16], in canine muscle stimulated electrically [13] and in horses [32].

Plasma K concentrations during exercise depend on the following factors. 1) The efflux rate from active muscles, that depends on the amount released from each active cell during each action potential, the motor units' firing rate and the muscle mass involved. 2) The rate of reuptake, mainly by the muscle itself, which is dependent on the number of $\mathrm{Na}^{+}-\mathrm{K}^{+}$pumps, whose density is higher in fast-twitch than in slow-twitch fibres [13]. 3) Muscle perfusion and K distribution to other tissues. 4) The haemoconcentration with sweat losses. 
At the end of submaximal cycling exercise to exhaustion, Van Beaumont $e t$ al. [32] showed that twothirds of a $24 \%$ increase in plasma $\mathrm{K}$ levels was due to haemoconcentration and one-third was due to a true increase in plasma $\mathrm{K}$ content. 5) $\mathrm{K}$ release from the erythrocytes, which may reflect a decrease in erythrocyte $\mathrm{K}$ concentrations or intravascular haemolysis, as was found in endurance, horses [2] and marathon athletes [26].

Increased K concentrations could enhance the activity of some enzymes involved in membrane transport, as well as increasing the oxygen consumption and the availability of intracellular calcium for release during activation of muscle membranes [14]. Moreover, $\mathrm{K}$ is important in neuromuscular function and it is possible that the balance between K efflux and influx could affect both contractile and metabolic properties of working muscle. A misbalance with an accumulation of $\mathrm{K}$ in the intersticial space between muscle fibres or in the extracelular space in the transverse-tubular system can reduce the membrane potential, leading to fatigue and eliciting cardiac arrhythmias $[11,15,17]$.

During training the density of $\mathrm{Na}^{+}-\mathrm{K}^{+}$pumps in skeletal muscle increased $[4,18]$, although the best training stimulus is unknown. Medbo and Sejersted [19] reported that sprint-trained subjects have higher K concentrations both at rest and during exercise and recovery than endurance-trained subjects. More recently it has been reported that a short training period of moderate intensity results in attenuation of the increase in plasma K during high intensity exercise. This finding was consistent with a decrease in K efflux from working muscles during exercise caused by an increase in skeletal muscle Na-K-ATPase [17].

We have previously demonstrated that significant differences in the adaptation to determined exercise intensity exist between Andalusian and other equine breeds. Andalusian horses had a higher heart rate, haemoglobin concentration, packed cell volume and plasma lactate values after a standardised exercise test than Angloarabian (AA) horses [22]. These differences were related to muscle composition, the enzyme profile and locomotor pattern [1, 21, 27]. Gluteus medium muscle of both untrained and trained Andalusian horses contains more type I fibres and less type IIA fibres than AA horses [27]. As for the enzyme profile, Andalusian horses have higher activities of lactate dehydrogenase (LDH) and glycogen phosphorylase (PHOS), and lower citrate synthetase (CS) and 3-0H- acyl coenzyme A dehydrogenase (HAD) than AA horses [1]. In locomotion, two phases can be considered in a stride, a forward and an upward phase, which are called respectively the horizontal and the vertical components of the stride. The Andalusian breed is characterised by a greater stride vertical component, a finding that was related partially to longer shoulders, forearm and metacarpus [21]. Recently, it has been confirmed that the interbreed differences in the adaptations to exercise are not only dependent on the training programmes, since they persisted after a similar training protocol, although it seems that Andalusian horses need more training time to show signs of systemic adaptations [20].

Since most of these differences have the ability to modify plasma $\mathrm{K}$ levels, this study aims to describe plasma $\mathrm{K}$ changes induced by exercise in Andalusian and Anglo-Arabian horses. The systemic response to exercise is influenced by the fitness level. For this reason, a comparison has been made of the $\mathrm{K}$ response to exercise when the differences in the fitness level were less marked, i.e., after training in Andalusian and before training in Angloarabian horses. Furthermore, the influence of two training programmes on plasma $\mathrm{K}$ levels has been assessed. As training was different in the two breeds studied, the relationship between $\mathrm{K}$ and exercise intensity was evaluated. In order to avoid interbreed differences linked to the degree of physical fitness, the relative exercise intensity was expressed as cardiovascular work and glycolytic response.

\section{Material and Methods}

\section{Horses}

A total of 30 animals, 20 Andalusian (AN) and 10 Angloarabian (AA), aged between 3 and 4 years, were studied. The AN horses were stallions, 9 AA were female and 3 were stallions. No special criteria were followed in selecting these animals, although they were subjected to the same diet, handling procedures and vaccination and deworming schedules. Prior to the tests, careful respiratory, cardiovascular and locomotor examinations were carried out. Furthermore, routinely haematological and biochemical analyses were performed. The animals with poor performance or with any physical abnormality were eliminated from the study. Untrained horses during the last two weeks before starting the research because of lameness were not considered. 


\section{Training programmes}

AN horses: The pre-training or breaking-in period, lasting for 5 weeks, was composed of walking and trotting exercises for $20 \mathrm{~min} /$ day, 5 days/week. By this time, work duration was progressively increased $5 \mathrm{~min} /$ week to the $10^{\text {th }}$ week, and cantering and 5-10 min galloping exercises were introduced. After performing the first exercise test, the AN horses started a 12 weektraining programme, with daily walking (20-30 $\mathrm{min})$, trotting (25-30 min) and cantering and galloping (12$15 \mathrm{~min}$ ) exercises, 5 days/week. In this stage, the second exercise test was carried out. The total duration of the training programme was 22 weeks.

This protocol has been traditionally used at the Centre for the Selection and Training of AN horses at Jerez de la Frontera. It is intended to improve fitness before the stallions start their reproductive life and therefore it is of low intensity. Moreover, it has been shown that this reduced exercise intensity is enough to potentate aerobic capacity, since glycolytic response to exercise was significantly reduced after training [22].

AA horses: The training programme for jumping events for the AA horses was composed of two phases, with a total duration of 24 weeks. The phase A (8 weeks) aimed to improve physical fitness and movement coordination. Horses were exercised for $35-40 \mathrm{~min} /$ day, 5 days/week ( 5 min walking, $20 \mathrm{~min}$ trotting and $5-10$ min galloping). During phase B (16 weeks), the AA horses were selectively trained for jumping. They performed daily exercises by walking (10 $\mathrm{min}$ at the beginning and $5 \mathrm{~min}$ at the end of each session), trotting (20-30 $\mathrm{min})$ and galloping (15 $\mathrm{min})$ and 3 days/week, the animals were subjected to a jumping test with 15-20 jumps at heights between 0.80 and 1.30 $\mathrm{m}[21]$.

\section{Standardised exercise test (SET)}

The horses carried out SETs of increasing intensity preceded by a warming-up period at a trot velocity of 4 $\mathrm{m} / \mathrm{s}$, over a distance of $1,000 \mathrm{~m}$. The SET started after a 2 min-rest-period, and was composed of four workloads at 5, 6, 7 and $8 \mathrm{~m} / \mathrm{sec}$, covering $1,000 \mathrm{~m}$ at each velocity. A 2 min-rest-period was allowed between workloads in order to avoid fatigue. The procedure used to standardise the velocity has already been described [21, 22]. The time was chronometered and real velocities were $4.94 \pm 0.37,6.08 \pm 0.47,7.08 \pm 0.49$ and $8.13 \pm 0.33 \mathrm{~m} / \mathrm{s}$. A paired $\mathrm{T}$ test failed to demonstrate any significant difference between these real velocities and the expected ones (5, 6, 7 and $8 \mathrm{~m} /$ sec).

This SET was chosen after demonstrating that it induces a plasma lactate concentration higher than the anaerobic threshold in both trained and untrained AN and AA horses [21]. It therefore seems useful to assess plasma $\mathrm{K}$ changes linked to exercise and training.

Two SETs were made in both breeds. SET1 was carried out after a pre-training period in the AN horses (week $10^{\text {th }}$ ) and after phase A in the AA horses (week $\left.8^{\text {th }}\right)$. SET2 was performed after 12 (week $22^{\text {nd }}$ ) and 16 weeks (week $24^{\text {th }}$ ) of training for AN and AA horses respectively.

\section{Heart rate recording}

Heart rate (HR) was monitored throughout the SETs with a Heart Rate Meter (Polar Sport Heart Rate Tester), carried by the riders on the wrist. Data were stored every $5 \mathrm{sec}$ and then downloaded to a computer with an interface for later analysis. The mean HR in each exercise level was calculated after HR stabilisation. The maximum HR or peak value (HRmax) was also annotated for each workload.

\section{Blood samples}

Jugular venous blood samples were collected at rest, immediately after the warming-up period, within the first $30 \mathrm{sec}$ after each exercise load and at 2, 4, 6, 8, 10, 15 and $30 \mathrm{~min}$ of an active recuperation at walking speed (approximate velocity $2.5 \mathrm{~m} / \mathrm{sec}$ ).

Blood was divided into two fractions with EDTA-3K and heparin-lithium. The first was used for haematological analysis and the second for plasma analysis after rapid centrifugation to avoid ion shifts thorough erythrocyte membranes and glycolytic processes. Both blood and plasma samples were kept on ice until their analysis, which was done within $12 \mathrm{hr}$ after extraction.

\section{Blood and plasma analysis}

Packed cell volume (PCV) was measured with a semiautomatic analyser (Sysmex-F820). Plasma lactate (LA) was quantified by an enzymatic method (Analox Champion PLM5), based on the pyruvate reduction by the L-lactate oxidoreductase. Plasma potassium levels (K) and total plasma protein (TPP) were measured with an analyser with selective electrodes for electrolytes (Ciba-Corning 644) and a refractometer (Atago) respectively. 


\section{Functional indices}

To assess physical shape, four functional indices were calculated. VLA2 and VLA4 were the velocities at plasma lactate levels of 2 and $4 \mathrm{mmol} / l$, respectively. They were obtained after extrapolation in the exponential relationship between plasma lactate levels (OY axis) and exercise velocity (OX axis). V150 and V200 represent the velocities at heart rates of 150 and 200 bpm respectively. They were obtained by extrapolation in the linear relationship between heart rate (OY axis) and exercise velocity (OX axis).

\section{Filming procedures and image processing}

During the SETs, the horses were filmed from the left side with a magnetoscopic camera (Sony, Video Camcorder Hi8 Handycam CCD-TR7050E), placed perpendicular to the direction of the plane of motion of the animals. The camera was placed at a sufficient distance to record field views of $25 \mathrm{~m}$, in order to ensure that the horses would execute at least 5 to 6 trot strides and 4 to 5 gallop strides. Furthermore, the camera was positioned halfway along the track, so the horses had time to stabilise their stride pattern before filming.

Velocity and temporal measurements of each image were calculated with a full-motion digital video overlay and video capture board (Video Blaster SE100, Creative labs). Lineal parameters were obtained from the relationships between the temporal variables and the velocity, by the procedure described in detail by Muñoz et al. [21, 22]. All the results are presented as the mean of 5 strides at a trot and 3 or 4 at a gallop in order to guarantee reproducibility of the locomotor pattern [22].

\section{Locomotor pattern}

The following parameters were included: stride duration (SD, s, the interval between two successive contacts of the same hoof); stride frequency (SF, strides/min, the number of strides per unit of time) and stride length (SL, m, the lineal hoof advancement during one stride).

\section{Statistical analysis}

The differences between exercise levels for each breed, as well as the changes induced by training, have been investigated by a t-test for dependent variables. A linear correlation (Pearson product - moment correlation) was used to analyse the relationships between plasma K and HR, PCV, LA, TPP, SD, SF and
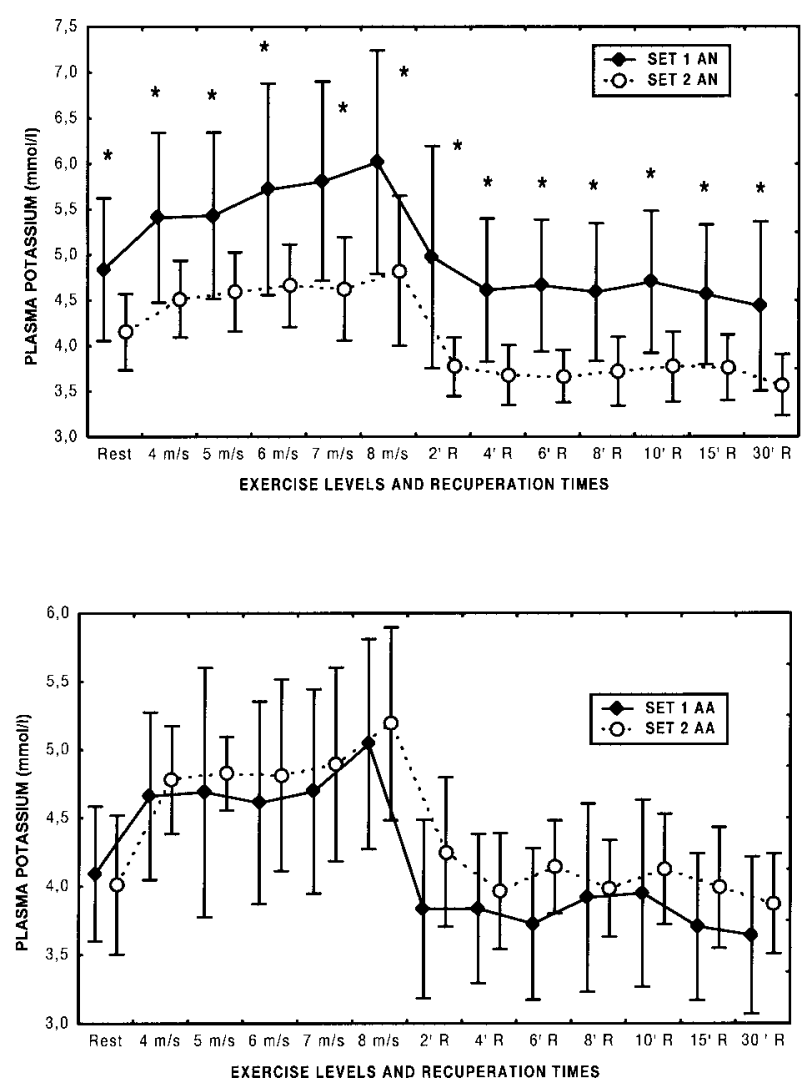

Fig. 1. Mean ( \pm SD) plasma potassium concentrations in Andalusian (O) and Angloarabian ( $\bigcirc)$ horses during the standardised exercise test. Differences are marked at $\mathrm{p}<0.05$.

SL. All the statistical analysis results were considered significant at $\mathrm{p}<0.05$.

\section{Results}

Data are presented as the mean $\pm \mathrm{SD}$ (otherwise indicated). The evolution of the plasma $\mathrm{K}$ concentrations with the SETs was similar in both breeds. K increased over resting values from $6 \mathrm{~m} / \mathrm{sec}$ in the $\mathrm{AN}$ and from $8 \mathrm{~m} / \mathrm{sec}$ in the AA horses. Plasma $\mathrm{K}$ decreased from exercise values after $2 \mathrm{~min}$ of an active recuperation in both breeds (Fig. 1). The effects of training on plasma $\mathrm{K}$ were more evident in the AN horses, with a smaller increase in plasma $\mathrm{K}$ concentrations after the different phases of the SET (rest, warming-up, workloads and recuperation period). But no such differences were detected in the AA horses (Fig. 1). 
Table 1. Mean ( \pm SD) of the functional indices in Andalusian and Angloarabian horses before and after training (V150 and V200: velocities at heart rates of 150 and $200 \mathrm{bpm}$; VLA2 and VLA4: velocities at plasma lactate concentrations of 2 and $4 \mathrm{mmol} / l$ )

\begin{tabular}{|c|c|c|c|c|}
\hline & \multicolumn{2}{|c|}{ Andalusian horses } & \multicolumn{2}{|c|}{ Angloarabian horses } \\
\hline & SET AN 1 & SET AN 2 & SET AA 1 & SET AA 2 \\
\hline $\mathrm{V}_{\mathrm{LA} 2}(\mathrm{~m} / \mathrm{s})$ & $4.187 \pm 0.91 * * *$ & $4.349 \pm 0.80^{* * * *}$ & $5.696 \pm 1.08$ & $6.043 \pm 1.24$ \\
\hline $\mathrm{V}_{\mathrm{LA} 4}(\mathrm{~m} / \mathrm{s})$ & $6.056 \pm 1.04^{* * * *}$ & $6.445 \pm 0.95^{* * * *}$ & $8.013 \pm 0.96^{\text {IT }}$ & $8.590 \pm 1.90$ \\
\hline $\mathrm{V}_{150}(\mathrm{~m} / \mathrm{s})$ & $5.156 \pm 0.36^{* * * *}$ & $5.214 \pm 0.50^{* * * *}$ & $6.286 \pm 0.65^{\mathbb{I}}$ & $6.143 \pm 0.82$ \\
\hline $\mathrm{V}_{200}(\mathrm{~m} / \mathrm{s})$ & $7.743 \pm 0.58 * * *$ & $7.804 \pm 0.63 * * *$ & $9.246 \pm 1.00^{\mathbb{I}}$ & $8.886 \pm 1.23$ \\
\hline
\end{tabular}

***: Differences between SET1 AN-AA and SET2 AN-AA, p<0.001, I: Differences between SET2 AN - SET1 AA, p<0.05, II: Differences between SET2 AN - SET1 AA, p<0.01.

Table 2. Mean values $( \pm$ SD) for the HR, PCV, LA, TPP, SF, SL and SD immediately after exercise, before and after training, in Andalusian and Angloarabian horses

\begin{tabular}{llccc}
\hline & \multicolumn{2}{c}{ Andalusian horses } & \multicolumn{2}{c}{ Angloarabian horses } \\
& SET AN 1 & SET AN 2 & SET AA 1 & SET AA 2 \\
\hline HRO' (bpm) & $206.2 \pm 6.27 * * *$ & $195.7 \pm 11^{* * *}$ & $181.3 \pm 11.6$ & $175.8 \pm 29$ \\
PCVO' (\%) & $57.89 \pm 3.89 * *$ & $51.9 \pm 4.20$ & $51.68 \pm 4.54$ & $50.32 \pm 2.90$ \\
LAO' (mmol/l) & $14.88 \pm 4.26^{* *}$ & $11.10 \pm 3.2^{* *}$ & $9.146 \pm 5.29$ & $7.482 \pm 4.8$ \\
TPP (g/d $l$ I & $7.067 \pm 0.36$ & $6.480 \pm 0.39$ & $6.936 \pm 0.415$ & $6.925 \pm 0.31$ \\
SFO' (stride/s) & $2.003 \pm 0.13^{* *}$ & $2.031 \pm 0.20^{*}$ & $1.831 \pm 0.20^{\mathbb{I}}$ & $1.992 \pm 0.15$ \\
SLO' (m/stride) & $2.035 \pm 0.21^{* * *}$ & $2.025 \pm 0.34^{* * *}$ & $4.017 \pm 0.31^{\mathbb{I} \text { II }}$ & $4.557 \pm 0.68$ \\
SDO' (s/stride) & $0.501 \pm 0.03^{* * *}$ & $0.493 \pm 0.03^{*}$ & $0.497 \pm 0.05$ & $0.502 \pm 0.35$ \\
\hline
\end{tabular}

***: Differences between SET1 AN-AA and SET2 AN-AA, p<0.001, **: Differences between SET1 and AN-AA y and SET2 AN-AA, p<0.01, *: Differences between SET1 AN-AA and SET2 AN-AA, p $<0.05$, IIIII: Differences between SET2 AN - SET1 AA, p<0.001, III: Differences between SET2 AN - SET1 AA, $\mathrm{p}<0.01$, II: Differences between SET2 AN - SET1 AA, $\mathrm{p}<0.05$.

In order to minimise the effects of the different interbreed fitness levels in the metabolic response to exercise, the SET2 for AN horses was compared to the SET1 for AA horses. The values of the four functional indices, VLA2, VLA4, V150 and V200 are shown in Table 1 for both horse breeds in SET1 and SET2. The values for $\mathrm{HR}, \mathrm{PCV}, \mathrm{LA}, \mathrm{TPP}, \mathrm{SF}, \mathrm{SL}$ and $\mathrm{SD}$ immediately after exercise (o') are shown in Table 2. Figure 2 shows the differences in HR, PCV and plasma LA between the SET2 for AN and the SET1 for AA horses. AN horses had higher HR values for the different test workloads, as well as at 2 and $15 \mathrm{~min}$ of recuperation. Similarly, the plasma LA accumulation was higher in AN horses from $5 \mathrm{~m} / \mathrm{s}$, without any significant differences during the recovery period (Fig. 2).

The mean values for plasma K, TPP and SL are shown in Fig. 3. The plasma K concentrations were higher in AN horses at rest, during exercise and during the $30 \mathrm{~min}$ of active recuperation. No interbreed differences were obtained in TPP during exercise, but during the first $10 \mathrm{~min}$ of the recuperation period, the TPP concentrations were significantly higher in the AN group. Finally, the AA horses were characterised by having higher SL both at trotting ( 4 and $5 \mathrm{~m} / \mathrm{sec}$ ) and at galloping $(6,7$, and $8 \mathrm{~m} / \mathrm{sec})$ velocities.

The correlations between plasma $\mathrm{K}$, metabolic response to exercise and locomotor pattern are shown in Tables 3 and 4, for AN and AA horses respectively.

\section{Discussion}

\section{Exercise effects in $A N$ and $A A$ horses}

Although both breeds were subjected to the same absolute workload, the relative intensity significantly differs, and therefore the interbreed differences must be interpreted with caution. The relative intensity of an exercise can be calculated from the following expression: \%VO2max $=1.617 \% \mathrm{HRmax}-65.02$, where VO2max is the maximum oxygen uptake and HRmax the maximum heart rate during exercise [6]. 

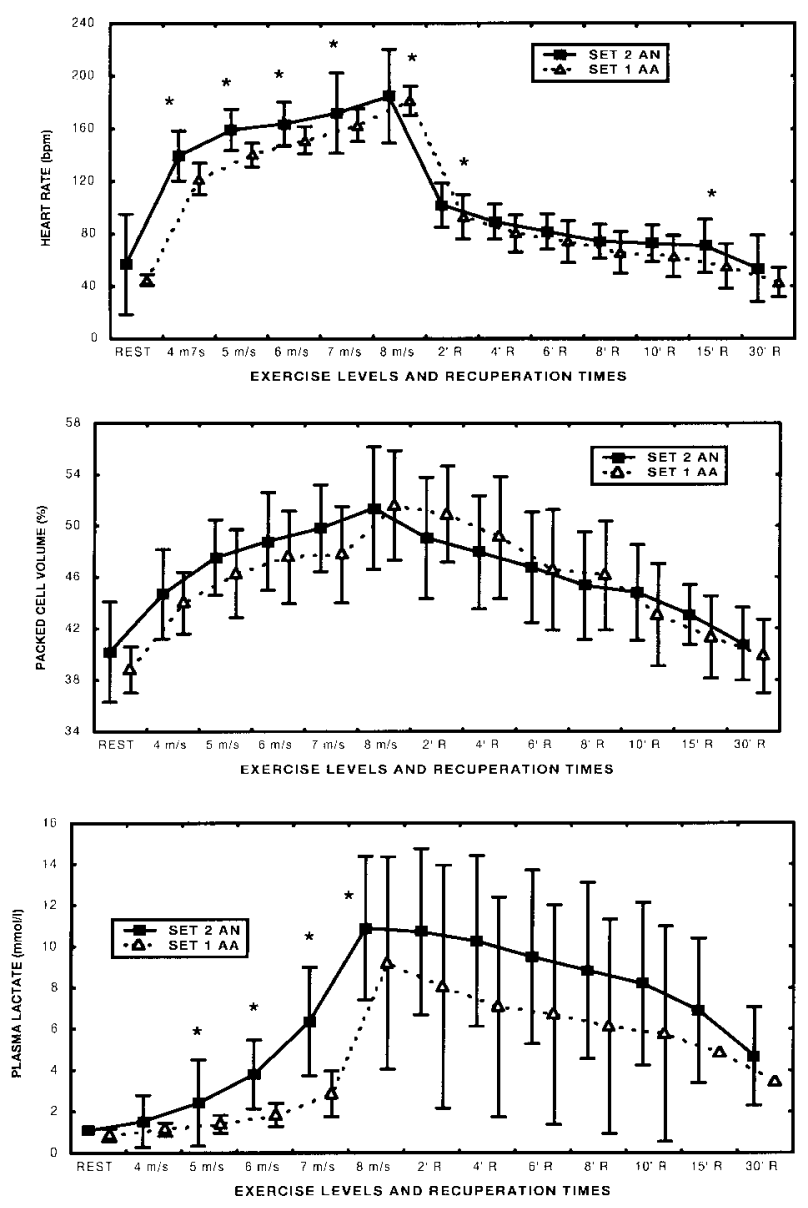

Fig. 2. Mean values $( \pm \mathrm{SD})$ for heart rate, packed cell volume and plasma lactate in Andalusian ( $\boldsymbol{\square}$ ) and Angloarabian $(\triangle)$ horses during the standardised exercise test. Differences are marked at $\mathrm{p}<0.05$.

The individual HRmax for these animals was unknown, since the SET was not performed at maximal velocity, but according to our previous results, in both breeds, a mean of $230 \mathrm{bpm}$ can be assumed [21, 22]. With the former expression, the AN horses would have been exercised at 79.94 and $72.57 \%$ and the AA horses at 62.37 and $58.76 \%$ VO2max, before and after training respectively.

The trend followed by the plasma K during the SETs was similar in the two breeds. The pattern observed is typical of prolonged submaximal physical activities, with an increase during the first exercise levels, a steady-state during the following exercise loads and a rapid decrease towards resting values after 2 min of active recuperation [31]. By contrast, during a brief high intensity exercise, there is a progressive increase
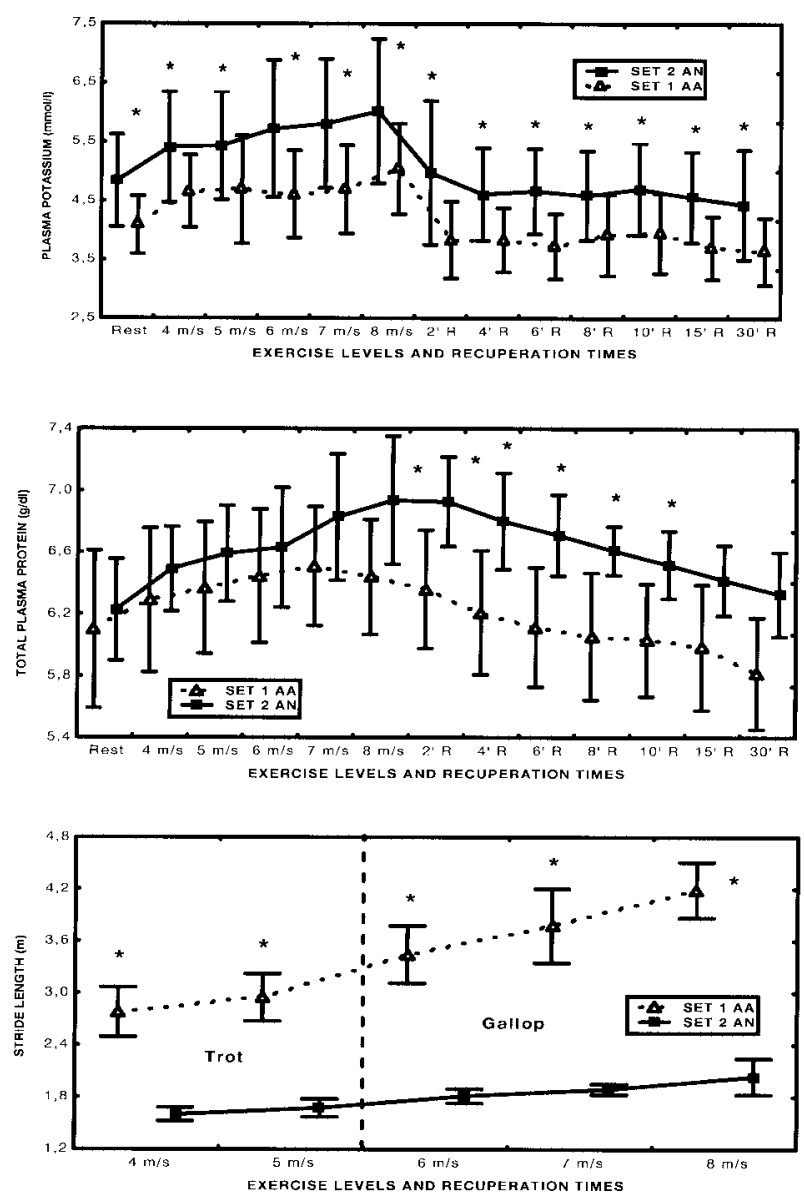

Fig. 3. Mean values $( \pm S D)$ for plasma potassium, total plasma proteins and stride length in Andalusian ( $\square$ ) and Angloarabian $(\triangle)$ horses during the standardised exercise test. Differences are marked at $\mathrm{p}<0.05$.

in relation to work intensity $[7,11]$.

The steady state of the plasma $\mathrm{K}$ concentrations from $6 \mathrm{~m} / \mathrm{sec}$ in $\mathrm{AN}$ and $8 \mathrm{~m} / \mathrm{sec}$ in AA horses to the end of the SET suggests that the release from muscle matched the reuptake, the intercompartimental fluid shifts due to hydrostatic (increased filtration pressure) and osmotic factors (hyperosmolality in muscle tissue) and thermoregulatory processes (lost in sweating and transpiration across respiratory mucoses). Therefore, the effect of dehydration must be considered. Toll et al. [30] described a method for calculating the decrease in plasma volume (PV) during exercise, after assuming that the total quantity of protein in the plasma compartment remained constant during exercise. Implicit in this assumption is that the protein molecules are too large to be forced out of the 
Table 3. Correlation coefficients for cardiovascular, metabolic and locomotor adaptations to exercise in untrained and trained Andalusian horses

\begin{tabular}{lccccccc}
\hline & HR & PCV & LA & K & TPP & SL & SD \\
\hline PCV & $0.710^{*}$ & & & & & & \\
LA & $0.570^{*}$ & $0.600^{*}$ & & & & & \\
K & $0.420^{*}$ & $0.530^{*}$ & n.s. & & & & \\
TPP & $0.420^{*}$ & $0.590^{*}$ & $0.300^{*}$ & $0.370^{*}$ & & & \\
SL & $0.950^{*}$ & $0.790^{*}$ & $0.610^{*}$ & $0.400^{*}$ & $0.530^{*}$ & & \\
SD & $0.800^{*}$ & $0.500^{*}$ & $0.160^{*}$ & $0.270^{*}$ & $0.370^{*}$ & $0.800^{*}$ & \\
SF & $0.940^{*}$ & $0.700^{*}$ & $0.460^{*}$ & $0.370^{*}$ & $0.460^{*}$ & $0.960^{*}$ & $0.900^{*}$ \\
\hline
\end{tabular}

Table 4. Correlation coefficients for cardiovascular, metabolic and locomotor adaptations to exercise in untrained and trained Angloarabian horses

\begin{tabular}{lccccccr}
\hline & HR & PCV & LA & K & TPP & SL & SD \\
\hline PCV & $0.610^{*}$ & & & & & & \\
LA & $0.610^{*}$ & $0.280^{*}$ & & & & & \\
K & $0.340^{*}$ & $0.520^{*}$ & $0.290^{*}$ & & & & \\
TPP & $0.551^{*}$ & $0.360^{*}$ & $0.190^{*}$ & $0.610^{*}$ & & & \\
SL & $0.960^{*}$ & $0.520^{*}$ & $0.610^{*}$ & $0.190^{*}$ & $0.460^{*}$ & & \\
SD & $0.960^{*}$ & $0.520^{*}$ & $0.610^{*}$ & $0.210^{*}$ & $0.410^{*}$ & $0.860^{*}$ & \\
SF & $0.950^{*}$ & $0.620^{*}$ & $0.460^{*}$ & $0.340^{*}$ & $0.470^{*}$ & $0.930^{*}$ & $0.890^{*}$ \\
\hline
\end{tabular}

capillaries during exercise, that no proteins are added and no notable degradation or production occurs. Some studies have indicated that some protein may be lost during exercise [5]. If this was the case, the changes in PV have been $25 \%$ underestimated, although this fact, according to the results of Toll et al. [30], does not substantially modify the results.

The calculation of the reduction of PV during exercise, expressed as a percentage of resting total blood volume (TBV) has been made as indicated for the AN breed:

PVo = 1 - PCVo' (PVo: resting plasma volume; PCVo: resting packed cell volume)

PVo $=1-0.399: 60.1 \%$ of TBV (since TBV: PV + $\mathrm{RBCV}$, where RBCV is the red cell volume)

The modifications in PCVo induced by exercise were calculated from the TPP values, following these indications:

Change in PVo':

(TPPo-TPPo') / TPPo' (PVo': post-exercise plasma volume; TPPo: resting total plasma proteins; TPPo': post-exercise total plasma proteins)

Change in PVo' in AN horses:

$(6.042-7.477 \mathrm{mg} / \mathrm{d} l) / 7.477 \mathrm{mg} / \mathrm{d} l=-0.067$

PVo' $=\mathrm{PVo} x$ change in PVo' $+\mathrm{PVo}$

$\mathrm{PVo}^{\prime}=0.601 \times(-0.067)+0.601=0.561$
Therefore:

$\mathrm{PVO}=60.1 \% \mathrm{TBV}$

PVo' $=56.1 \% \mathrm{TBV}$

These calculations were also carried out for AA horses and these values were found: $\mathrm{PVo}=60.9 \% \mathrm{TBV}$ and $\mathrm{PVo}^{\prime}=56.8 \%$ TBV.

These decreases in PV (4\% in AN and $2.1 \%$ in AA horses) might influence the hyperkalemia observed during exercise in the two breeds. Furthermore, the haemoconcentration would have also been related to the magnitude of the splenic mobilisation, which is proportional to the oxygen requirements in active tissues as muscles, up to when the spleen is maximally contracted [22, 24, 25]. The red blood cell volume (RBCV) can be calculated as a function of the resting TBV, using the PCV $[24,30]$. According to these equations, the RBCV could account for $43.4 \%$ and $41.2 \%$ of the TBV in the $\mathrm{AN}$ and $\mathrm{AA}$ horses after exercise $(39.9 \%$ and $39.1 \%$ at rest in $\mathrm{AN}$ and $\mathrm{AA}$ horses). In conclusion, although blood mobilisation from the spleen and haemoconcentration influenced the hyperkalemia observed during exercise in both equine breeds, the main factor was the efflux from the working muscles, since PCV returned to baseline values after 15 min of recuperation, as confirmed by the correlation analysis. The higher haemoconcentration in AN horses did not result from the climatic 
conditions. Although the SETs were carried out on different days, an ambient impact factor was obtained by adding the mean temperature $\left({ }^{\circ} \mathrm{F}\right)$ and the relative humidity (\%). This index ranged between 125.3 and 131.0 and an ANOVA did not reveal any significant difference between the days when the SETs were performed in the two breeds. It has been suggested that the greater sweating rate in AN horses was of concern in these results, but it has not been proven yet [21].

It has been reported that muscle $\mathrm{K}$ release during exercise is partially determined by the amount of active mass, so the body size should be considered, but the withers' height was $159.9 \pm 2.25$ and $160.5 \pm 2.13 \mathrm{~cm}$ in AN and AA horses respectively.

Previous researches have shown that the locomotion pattern may be partly dependent on both muscle composition and metabolic profile [21, 22]. In fact, SF has been related to the amount of $\mathrm{K}$ released by muscle fibre. In the present work, AN and AA horses had a different locomotor pattern, with longer SL in the latter breed, and therefore, lesser SF at a defined velocity, in agreement with previous results. But these differences did not seem to generate any significant difference in the exercise-induced hyperkalemia. On the other hand, the influence of rate muscle firing cannot be omitted.

A surprising result was the lack of significant correlations between LA and K during exercise in SET1 of AN horses. Some researches have separated the effects of blood acidosis on hyperkalemia [3]. Other possible explanations are the reduction of muscle LA clearance by a reduced blood supply secondary to hypovolemia and/or a limitation in the muscle - plasma diffusion rate, after reaching high plasma LA levels [23].

\section{Effect of training in $A N$ and $A A$ horses}

Since training programmes were different, no interbreed differences could be analysed.

The reduction of effort-induced hyperkalemia after training in AN horses could represent a lesser physical stress in response to the same exercise intensity. The exercise hypovolemia was less evident after training in this breed (PCV of $57.98 \%$ in SET1 and $51.90 \%$ in SET2). It has been reported that increases in PCV during physical activities increase the erythrocyte membrane surface for ion exchange in whole blood. This fact could also been implicated in the reduced hyperkalemia after training, although no conclusion can be withdrawn, since only plasma $\mathrm{K}$ has been considered. Moreover, other factors, such as a change in locomotion, and an increase in the density and functionality of the Na-K ATPase pump should also be related to these results.

A training programme similar to that described in the present report showed an increase in the oxidative potential and percentage of IIA fibres together with a decrease in IIB fibres and I and IIA area fibres in the inner parts of the gluteus medium muscle [27]. Although these adaptations are advantageous from a metabolic point of view (smaller distance for oxygen and metabolite diffusion), they imply a reduction in strength, since a positive correlation exists between fibre size and contractile proteins [12]. The reduction in the glycolytic response to the SET2 represents a more intense aerobic metabolism, more dependent on I and IIA fibres, and therefore less muscle acidosis and $\mathrm{K}$ release can be expected.

Most authors have reported a longer stride length after training $[9,10]$, but the opposite has been described in AN horses [21, 22]. The reduced plasma $\mathrm{K}$ response to exercise in spite of this adaptation to training might indicate that the number of contractions per unit time was not the major determinant of the kalemia. It seems more likely that stride length and frequency are important in determining the initial rise in $\mathrm{K}$ and thereafter other factors are crucial.

Two hypotheses can be proposed to explain the lack of $\mathrm{K}$ changes after training in the AA breed. First, it might be thought that cardiovascular and metabolic adaptations occurred at the beginning of the training (phase A), since no differences in HR, LA, PCV or $\mathrm{Hb}$ were observed between SET1 and SET2 in this breed. Secondly, training for jumping implies a development of strength, being energetically dependent on anaerobic metabolism. If the active muscles have released more $\mathrm{K}$ after training, then more $\mathrm{K}$ would have been reuptaken by $\mathrm{Na}^{+}-\mathrm{K}^{+}$ATPase pumps, more developed in II fibres, as has been demonstrated after a short-term training [17].

In conclusion, the main determining factor in exercise-induced hyperkalemia in the two equine breeds studied seems to be the relative intensity of the effort. The improvement in fitness in AN horses, with a trend towards a more oxidative metabolism, reduced the degree of hyperkalemia. In spite of the longer SL of both untrained and trained AA horses, the locomotor pattern has the same effect on plasma $\mathrm{K}$ 
accumulation in both breeds. Therefore, plasma K levels might indicate the degree of physical stress undergone by $\mathrm{AN}$ and $\mathrm{AA}$ horses during exercise and training.

\section{Acknowledgments}

This study has been supported by the Andalusian Research Council (Group AGR-0111) and a fellowship form the University of Córdoba. The authors are very grateful to the staff, riders and trainers of the Centres of Selection and Horse Training of the Military Stud of Jerez de la Frontera and Ecija (Spain), who willingly cooperated in the performance of the exercise test and training programme.

\section{References}

1. Blanco, L. 1995. Adaptaciones bioquímicas del músculo esquelético debidas al entrenamiento en caballos de raza Española y Arabe. PhD Thesis, University of Córdoba, Spain.

2. Boucher, J.H., Ferguson, E.W., Wilhelmsen, C.L., Statham, N., and McMeek, R. 1981. Erythrocyte alterations during endurance exercise in horses. $J$. Appl. Physiol. 51, 131-134.

3. Busse, M.W. and Maasen, N. 1989. Effect of consecutive exercise bouts on plasma potassium concentration during exercise and recovery. Med. Sci. Sports Exerc. 21, 489-493.

4. Clausen, T. 1990. Significance of $\mathrm{Na}^{+}-\mathrm{K}^{+}$pump regulation in skeletal muscle. N.I.P.S. 5, 148-151.

5. Coyne, C.P., Carlson, G.P., Spensley, M.S., and Smith, J. 1990. Preliminary investigation of alterations in blood viscosity, cellular composition, and electrophoresis plasma protein fraction profile after competitive racing activity in Thoroughbred horses. Am. J. Vet. Res. 51, 19561963.

6. Evans, D.L. and Rose, R.J. 1988. Determination and repeatibility of maximal oxygen consumption and other cardiorespiratory measurements in the horse. Equine Vet. J. 20, 94-98.

7. Ferrante, P., Taylor, L.E., Wilson, J.A., and Kronfeld, D.S. 1995. Plasma and erythrocyte ion concentrations during exercise in Arabian horses. Equine Vet. J. 18, 306-309.

8. Fosha-Dolezal, S.R. and Fedde, M.R. 1988. Serum potassium during exercise in Hereford calves: influence of physical conditioning. J. Appl. Physiol. 65, 1360-1366.

9. Gottlieb-Vedi, M., Persson, S., Erickson, H., and Korbutiak, E. 1995. Cardiovascular, respiratory and metabolic effects of interval training at VLA4. J. Vet. Med. A. 42, 165-175.

10. Harkins, J.D. 1992. The effect of detraining on performance in equine athletes: a review. In: Assoc. Eq. Sports Med. Proc. 57-58.

11. Harris, P.A. and Snow, D.H. 1992. Plasma potassium and lactate concentrations in Thoroughbred horses during exercises of varying intensity. Equine Vet. J. 23, 220-225.

12. Hill, A.V. 1950. The dimensions of animals and their muscular dynamics. Sci. Prog. 38, 209-229.

13. Hirche, H., Schumacher, E., and Hagemann, H. 1980. Extracellular $\mathrm{K}^{+}$concentration and $\mathrm{K}^{+}$ balance of the gastrocnemius muscle of the dog during exercise. Pflügers Arch. 387, 231-237.

14. Hnik, P., Viskocil, F., Vejoada, R., Ujec, E., and Rehfeldt, H. 1985. In: Biochemistry of exercise (Saltin B. ed.), Human Kinetics Publishers. Champaign. Illinois. pp. 345-364.

15. Jeul, C. 1988. The effects of $\beta 2$-adrenoceptor activation on ion shifts and fatigue in mouse soleus muscle stimulated in vitro. Acta Physiol. Scand. 134, 209-216.

16. Lindinger, M.I. and Sjogaard, G. 1991. Potassium regulation during exercise and recovery. Sports Med. 11, 382-401.

17. McCutcheon, L.J., Geor, R.J., and Shen, H. 1999. Skeletal muscle $\mathrm{Na}^{+}-\mathrm{K}^{+}$-ATPase and $\mathrm{K}^{+}$ homeostasis during exercise: effects of short-term training. Equine Vet. J. 30, 303-310.

18. McKenna, M.J., Harmer, A.R., Fraser, S.F., and Li, L.J. 1996. Effects of training on potassium, calcium and hydrogen ion regulation in skeletal muscle and blood during exercise. Acta Physiol. Scand. 156, 335-346.

19. Mebdo, J.I. and Sjersted, O.M. 1994. Plasma K ${ }^{+}$ changes during intense exercise in endurancetrained and sprint-trained subjects. Acta Physiol. Scand. 151, 363-371.

20. Muñoz, A., Riber, C., Santisteban, R., Lucas R.G., and Castejón, F.M. 2002. Effect of training duration and exercise on blood-borne substrates, plasma lactate and enzyme concentrations in Andalusian, Angloarabian and Arabian horses. Equine Vet. J. 34, 245-251.

21. Muñoz, A., Santisteban, R., Rubio, M.D., Agüera, E.I., and Castejón F.M. 1999. Locomotor response to exercise in relation to plasma lactate 
accumulation and heart rate in Andalusian and Anglo-Arabian horses. Vet. Res. Communic. 23, 269284.

22. Muñoz, A., Santisteban, R., Rubio, M.D., Agüera, E.I., Escribano, B.M., and Castejón, F.M. 1998. Locomotor, cardiocirculatory and metabolic adaptations to training in Andalusian and AngloArabian horses. Res. Vet. Sci. 66, 25-31.

23. Muñoz, A., Santisteban, R., Rubio, M.D., Vivo, R., Agüera, E.I., and Castejón, F.M. 1996. Cinética del lactato plasmático durante el ejercicio y la recuperación en caballos Pura Raza Española (Summary in English). Med. Vet. 13, 183-190.

24. Persson, S.G.B. 1967. On blood volume and working capacity in horses. Acta Vet. Scand. 19, 1189.

25. Persson, S.G.B. 1997. Heart rate and blood lactate responses to submaximal treadmill exercise in the normally performing Standardbred trotter. Age and sex variations and predictability from the total red blood cell volume. J. Vet. Med. A. 44, 125-132.

26. Reinhart, W.H., Staubli, H., and Straub, P.W. 1983. Impaired red cell filterability with elimination of old red blood cells during a 100-km race. J. Appl. Physiol. 54, 827-830.

27. Rivero, J.L., Ruz, M., Serrano, A.L., and Diz, A.M. 1995. Effects of a 3-month endurance training programme on skeletal muscle histochemistry in Andalusian, Arabian and Anglo-Arabian horses. Equine Vet. J. 27, 51-59.

28. Rubio, M.D., Vivo, R., Agüera, E.I., Escribano,
B.M., Muñoz, A., and Castejón, F.M. 1995. Electrolyte changes observed in Andalusian and Arab horses subjected to training. In: Assoc. Equine Sports Medicine Proceedings. 14.

29. Sejersted, O.M., Vollestad, H.K., and Medbo, J.I. 1986. Muscle fluid and electrolyte balance during and following exercise. Acta Physiol Scand. 556, 119-127.

30. Toll, P.W., Gaehtgens, P., Neuhaus, D., Pieschl, R.L. and, Fedde, M.R. 1995. Fluid, electrolyte and packed cell volume shifts in racing greyhounds. Am. J. Vet. Res. 56, 227-232.

31. Valberg, S., Essén-Gustavsson, B., Lindholm, A., and Persson, S. 1989. Blood chemistry and skeletal muscle metabolic responses during and after different speeds and durations of trotting. Equine Vet. J. 21, 91-95.

32. Valberg, S., Essén-Gustavsson, B., Lindholm, A., and Persson, S.G.B. 1989. Blood chemistry and skeletal muscle metabolic responses during and after different speeds and durations of trotting. Equine Vet. J. 21, 91-95.

33. Van Beaumont, W., Strand, J.C., Petrofsky, J.S., Hipskind, S.G., and Greenleaf, J.E. 1973. Changes in total plasma content of electrolytes and proteins with maximal exercise. J. Appl. Physiol. 34, 102-106.

34. Vollestad, N.K., Hallén, J., and Sejersted, M. 1994. Effect of exercise intensity on potassium balance in muscle and blood of man. J. Physiol (London) 475, 335-368. 